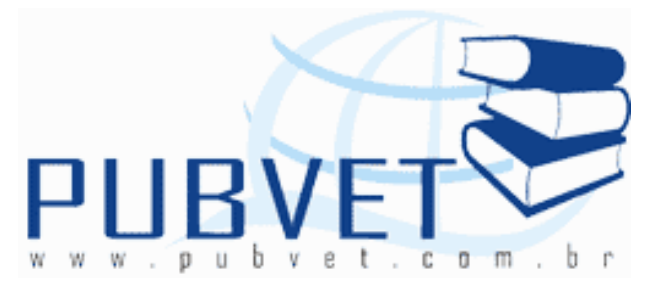

PUBVET, Publicações em Medicina Veterinária e Zootecnia.

\title{
Avaliação do perfil metabólico em vacas leiteiras de alta produção no período de transição
}

\section{Cristiane Francele Cupertino ${ }^{1}$, Evandro Pereira Neto ${ }^{2}$, Marcio Paiva Barcellos ${ }^{1}$,} Fabio Porto Sena ${ }^{3}$, Dominik Lenz ${ }^{4}$, Graziela Barioni (Orientadora) ${ }^{5}$

\author{
${ }^{1}$ Medico (a) Veterinário (a) Autônomo (a) \\ ${ }^{2}$ Mestrando em Ciência Animal - Centro Universitário Vila Velha \\ ${ }^{3}$ Técnico de Patologia do Centro Universitário Vila Velha \\ ${ }^{4}$ Professor do Centro Universitário Vila Velha \\ ${ }^{5}$ Professora da Universidade Federal do Espírito Santo
}

\section{Resumo}

Ao longo de décadas inovações em relação ao sistema de produção e a seleção racial na espécie bovina, tem sido aplicados com a finalidade de aumentar a produtividade leiteira. Essas mudanças no sistema de produção acarretam no aumento da demanda e exigência produtiva, favorecendo as doenças metabólicas, principalmente no período de transição, composto das três semanas antes do parto até as três semanas após o parto, período de maior predisposição as manifestações clínicas ou subclínicas. Por esse motivo, o presente trabalho teve como objetivo de estabelecer o perfil metabólico de magnésio, cálcio, fósforo, triglicerídeos e glicose de vacas leiteiras da raça holandesa durante o período de transição. Foram utilizadas 56 vacas da raça holandesa, com uma média de produção de 25 à $30 \mathrm{~kg} /$ dia de leite. As 
amostras foram coletadas em cinco momentos: 30 e 15 dias pré-parto, no dia do parto; 15 e 30 dias após o parto. Para dosagem bioquímica de cálcio constatou-se aumento significativo entre 30 dias do pré-parto e o parto. Aos 15 dias do pós-parto foi observada uma redução dos valores de cálcio, fósforo e magnésio em relação ao parto. No momento do parto constatou os maiores valores de glicose com diminuição dos triglicerídeos. Foi possível observar que mesmo sendo animais hígidos os valores oscilaram apresentando relevância estatística em relação ao parto. Dessa forma é de fundamental importância a avaliação do perfil metabólico no rebanho e manter uma dieta nutricional balanceada.

Palavras-chave: vacas, período de transição e perfil metabólico.

\section{Metabolic profile in dairy cows of high production in the transition period}

\section{Abstract}

For decades, innovations in relation to production system and racial selection in the bovine species, has been applied in order to increase milk productivity. The changes in the production system led to an increased amount of metabolic diseases, especially in the transition period, three weeks before calving until three weeks after childbirth, the period of highest susceptibility clinical or subclinical. Therefore, this study aimed to establish the metabolic profile of magnesium, calcium, phosphorus, triglycerides and glucose Holstein dairy cows during the transition period. 56 Holstein cows, with an average yield of 25 to $30 \mathrm{~kg}$ of milk per day were examined. The samples were collected in five time points: 30 and 15 days pre-partum, during birth, 15 and 30 days after delivery. The values of calcium showed a significant increase from 30 days before delivery and birth. At 15 days postpartum decreased levels of calcium, phosphorus and magnesium were observed. During birth the highest values of glucose in combination with a decrease in triglycerides were detected. It was observed that even though the values varied, healthy animals showed 
statistical significance in relation to delivery. Thus it is of fundamental importance to evaluate the metabolic profile in the herd and maintain a balanced nutritional diet.

Keywords: cows, transition period and metabolic profile.

\section{Introdução e Revisão de literatura}

A cadeia produtiva de leite é a mais importante do complexo agroindustrial Brasileiro, composta por um milhão de produtores de leite e produção aproximada de 20 bilhões de litros/ano, com grande potencial de abastecimento do mercado interno e externo. Movimenta anualmente aproximadamente 10 bilhões de dólares, empregando três milhões de pessoas. A produção nacional de leite cresceu aproximadamente 37\%, entre 1990 e 2000 (CARVALHO et al., 2002), isso pelo aumento do número de animais especializados, os quais atingem uma produtividade de 30 até 70 litros/vaca/dia, como afirmou (KOZLOSKI, 2002).

Com a finalidade de aumentar a produtividade leiteira, ao longo de décadas inovações em relação aos sistemas de produção e a seleção racial na espécie bovina, tem sido aplicados (KOZLOSKI, 2002). Essas mudanças acarretam no aumento da demanda e exigência produtiva, favorecendo o desequilíbrio entre disponibilidade dos nutrientes para o organismo, a capacidade de metabolização desses componentes e os níveis de produção alcançados desequilibrando a homeostase, favorecendo as doenças metabólicas (GONZÁLEZ, 2000; RADOSTITS, 2000).

De acordo com Radostits (2000), Wittwer (2000), Kozloski (2002) e Grummer (2009) as doenças metabólica são definidas como qualquer distúrbio que provoca disfunção da ação metabólica do organismo, resultando em perda do controle da homeostase, estas apresentam elevada prevalência e mortalidade. 
CUPERTINO, C.F. et al. Avaliação do perfil metabólico em vacas leiteiras de alta produção no período de transição. PUBVET, Londrina, V. 5, N. 18, Ed. 165, Art. 1115, 2011.

Portanto, alguns países têm desenvolvido sistemas para prever sua ocorrência e com isso minimizar as perdas econômicas.

O período de transição, composto das três semanas antes do parto até as três semanas do pós-parto, tem maior predisposição as manifestações clínicas ou subclínicas das enfermidades metabólicas, portanto é considerado uma fase de desafio na produção de vacas leiteiras. Nesta fase ocorrem diversas mudanças no sistema endócrino e metabólico que favorecem o desequilíbrio da homeostase (GRUMMER, 1995).

Huzzey et al. (2007) também ressaltam a ocorrência de várias alterações metabólicas, fisiológicas e anatômicas na vaca parturiente, as quais favorecem a ocorrência de diversas enfermidades resultando em prejuízo pela redução da produção leiteira e do desempenho reprodutivo, além do aumento da taxa de descarte.

Dentre as alterações fisiológicas e anatômicas que ocorrem no período de transição merecem destaque, ação hormonal, como a resistência a insulina, que segundo Petterson et al. (1993) em vacas leiteiras ocorre uma diminuição na concentração plasmática de insulina no período de transição. A secreção de insulina é dependente dos níveis plasmáticos de cálcio e glicose, no período de transição estes estão sendo desviados para o crescimento fetal e para a produção de colostro, ocorrendo assim uma diminuição na secreção de insulina, com isso ocorre menor capacidade de utilização de glicose como fonte energética. Essa condição irá interferir no organismo do animal, predispondo-o a distúrbios metabólicos (KOZLOSKI, 2002).

Outro hormônio responsável por alterações fisiológicas é o estrógeno que apresenta no terço final da gestação aumento na concentração plasmática, acredita-se que essa elevação está relacionado à inapetência sofrida pelas vacas nos dias que antecedem o parto, esse aumento faz com que haja uma 
CUPERTINO, C.F. et al. Avaliação do perfil metabólico em vacas leiteiras de alta produção no período de transição. PUBVET, Londrina, V. 5, N. 18, Ed. 165, Art. 1115, 2011.

maior lipomobilização durante os últimos dias de gestação, predispondo o fígado gorduroso, fator que predispõe a ocorrência de doenças como a cetose (CHEW et al., 1979).

Durante o período de transição a capacidade ruminal é alterado sendo um fator físico de grande importância para alterações fisiológicas do animal, o tamanho do feto no final da gestação aumenta a pressão interna nos órgãos digestivos, reduzindo a capacidade de armazenamento do rúmen, intensificando ainda mais o balanço energético negativo (BEN), que prejudica a absorção de nutrientes, sais minerais e a produção de ácidos graxos voláteis. Dessa forma ocorre a lipólise, para repor suas necessidades energéticas, que são demandadas em grande quantidade para o crescimento fetal e pela glândula mamária para a produção de colostro (CHEW et al., 1979; NIELEN et al., 1994).

No pós-parto, próxima fase do ciclo produtivo, ocorre o início súbito de uma lactação profusa, a qual pode reduzir ainda mais as reservas de nutrientes, associado à capacidade de ingestão de alimento limitada nas primeiras semanas após o parto, acarretam em BEN, ou seja, uma maior utilização de nutrientes energéticos (minerais) para a mantença e produção comparado com o que é ingerido, resultando em utilização de reservas corpóreas para garantir a produção de leite (NIELEN et al., 1994; GOFF, 2006).

Portanto, vacas que já apresentam BEN acentuado no pré-parto, apresentam uma taxa de lipólise superior durante a lactação fazendo com que a doença metabólica ocorra (RADOSTITS, 2000).

A lipólise acentuada decorrente da limitada ingestão de alimento, aumenta a probabilidade de ocorrerem lesões hepáticas como à infiltração de gordura que pode afetar a concentração de outros componentes sanguíneos como a glicose, proteína total, albumina e uréia podendo estar diminuídas, que por sua vez, 
possibilitará a ocorrência de transtornos metabólicos (WEST, 1990; AMETAJ, 2005).

As doenças metabólicas como a hipocalcemia e a cetose tem relação com afeç̧ões comuns neste período como a mastite, paratuberculose, salmonelose, retenção placentária e deslocamento de abomaso (KIMURA e GOFF, 2009).

A hipocalcemia, hipofosfatemia e hipomagnesemia podem ocorrer sob diversos graus, desde deficiências severas a leves, perturbações subclínicas, sintomas não-específicos, crescimento lento, problemas de fertilidade, baixo rendimento da carcaça e pouca produção de leite. Deficiências leves ou moderadas também podem causar prejuízos econômicos sérios, porque reduzem a produtividade dos animais e constituem obstáculos à melhoria dos rebanhos (PEIXOTO et. al., 2005).

A grande demanda de cálcio após o parto e perda do cálcio no colostro, está diretamente relacionada a variações nas concentrações desse íon e o volume do leite secretado nessas vacas selecionadas geneticamente para alta produção, podendo ser um indicador poderoso dos distúrbios metabólicos e deve ser monitorado em vacas após o parto (NRC, 2001; RODRIGUES e GONZÁLEZ, 2004; DUFFIELD e LEBLANC, 2009).

A avaliação do cálcio é justificada pela gravidade das alterações clínicas associadas a sua redução, sendo assim é importante manter a homeostase do cálcio sanguíneo, que para vacas adultas é mantida em torno de 8,5-10 $\mathrm{mg} / \mathrm{dL}$, ou 8,7 a $11,4 \mathrm{mg} / \mathrm{dL}$ respectivamente segundo Goff (2000) e Rosol e Capen (1997), a fim de impedir que o nível de cálcio sanguíneo diminua levando a sintomatologias indesejadas.

Goff e Horst (1997), descrevem que níveis plasmáticos de cálcio menores que $9,0 \mathrm{mg} / \mathrm{dL}$ durante o período de transição, caracterizam uma hipocalcemia não 
acarretando em alterações clínicas contudo levando a queda na produção e perdas econômicas. Já Duffield e Leblanc, (2009), afirmam que mesmo níveis de cálcio próximos a $7,5 \mathrm{mg} / \mathrm{dL}$ a hipocalcemia é subclínica, ou seja dentro dessas concentrações não há manifestações clínica.

Rodrigues e González (2004) consideram que níveis críticos sanguíneos de cálcio de $6,0 \mathrm{mg} / \mathrm{dL}$, é incompatível com a motilidade normal do trato gastrointestinal, o que pode exacerbar a hipocalcemia ou até causar outros problemas metabólicos, como o deslocamento de abomaso devido a diminuição das concentrações de cálcio na musculatura lisa, sendo essa vital para a função normal do trato digestivo.

Os níveis séricos de fósforo, situa-se entre 4 e $8 \mathrm{mg} / \mathrm{dL}$. Este é absorvido no intestino, através do transporte ativo responsivo a 1,25-diidroxivitamina $D$. Que é estimulada quando há níveis plasmáticos muito baixos de fósforo, portanto, a eficiência absortiva intestinal do fósforo, pode ser regulada de forma ascendente durante períodos de deficiências (HERDT, 1999; GOFF, 2006).

Além disso, o paratormônio (PTH), que é secretado durante a deficiência de cálcio, promove o aumento da excreção renal e salivar do fósforo, podendo dessa forma ser nocivo para a manutenção das concentrações séricas normais de fósforo. Motivo pelo qual animais hipocalcêmicos tendem a tornarem hipofosfatêmicos (HERDT, 1999; RADOSTITS, 2000; GOFF, 2006).

Para a homeostasia do magnésio ( $\mathrm{Mg}$ ) o controle hormonal não é eficiente, portanto a manutenção dos valores de normalidade são mantidos principalmente por controle da ingestão e da excreção renal, ou seja, caso este seja absorvido exageradamente, a concentração se elevará acima do limiar renal para a reabsorção e o excesso é excretado na urina. Os níveis de $\mathrm{Mg}$ se 
elevam quando o PTH é liberado em resposta à hipocalcemia, pois este eleva o limiar renal tanto para cálcio quanto para Mg (GOFF, 2006).

A concentração plasmática de $\mathrm{Mg}$, ficam normalmente em torno de 1,8 e 2,4 $\mathrm{mEq} / \mathrm{L}$ (GOFF, 2006). Para Radostits et al., (2000), as concentrações séricas de magnésio variam de 1,7 a $3 \mathrm{mEq} / \mathrm{L}$, nos casos de hipomagnesemia subclínica podem ser observados valores frequentemente reduzidos para 1 a 2 $\mathrm{mEq} / \mathrm{L}$, e em casos de tetania os níveis podem diminuir para $1,2 \mathrm{mEq} / \mathrm{L}$. No entanto, alguns animais podem apresentar níveis séricos de magnésio diminuídos em até $0,4 \mathrm{mEq} / \mathrm{L}$ sem a doença clínica.

Segundo Goff, (2000), se concentração sérica de Mg nas vacas no periparto não apresentar-se em torno de $2,0 \mathrm{mEq} / \mathrm{L}$, sugere que a absorção de $\mathrm{Mg}$ na dieta é inadequada e que a hipomagnesemia pode estar contribuindo para a hipocalcemia no rebanho.

Em relação aos níveis séricos de glicose são mantidos normalmente entre $60 \mathrm{e}$ $80 \mathrm{mg} / \mathrm{dL}$ e são tolerados níveis de até $40 \mathrm{mg} / \mathrm{dL}$ durante dias eventualmente. No final da gestação, há utilização de grandes quantidades de glicose, devido o crescimento fetal, onde ocorre a gliconeogênese, para formação de glicogênio hepático e muscular (VASQUEZ-ANON et al., 1994; GONZÁLEZ, 1997; GOFF, 2006).

No momento do parto, a glicemia tem um aumento agudo devido ao aumento das concentrações de glucagon e glicocorticóides decorrentes do estresse que acarreta a redução dos estoques de glicogênio no fígado (GRUMMER, 1995).

Após o parto a glicemia diminui novamente, especialmente na primeira semana em vacas de alta produção devido à lactação impor grande demanda sobre o metabolismo da glicose onde pode levar a um quadro de hipoglicemia que pode não ser detectadas clinicamente o que indica uma ingestão 
insuficiente de energia (VASQUEZ-ANON et al., 1994; GONZÁLEZ, 1997; RADOSTITS et al., 2000 ; GOFF, 2006).

Apesar do nível de glicose não ser um bom indicador do nível energético da dieta, é uma molécula vital para as necessidades energéticas do organismo que justifica sua inclusão no perfil metabólico (GONZÁLEZ, 1997).

Além disso, devido às altas produções de leite no início da lactação que demandam quantidades elevadas de energia, ocorre a lipomobilização das reservas corporais da vaca para serem utilizadas como fonte energética. Neste período de transição, ocorre um acúmulo de triglicerídeos hepático de forma fisiológica. Mas quando o corre a lipomobilização, e a concentração de ácidos graxos não esterificados (AGNE) no fígado ultrapassa sua capacidade em oxidar, há maior acúmulo de triglicerídeos, comprometendo a função hepática (DUFFIELD e LEBLANC, 2009; GOZÁLEZ, 2009).

Assim sendo pode-se constatar que os níveis séricos de triglicerídeos aumentam em decorrência do BEN. Além do aumento da demanda de glicose para a produção de leite (SMITH et al., 1997; BOBE et al., 2004; DUFFIELD e LEBLANC, 2009). Segundo Kaneko et al. (1997), os valores séricos de triglicerídeos variam entre 0,4 a $14 \mathrm{mg} / \mathrm{dL}$ em vacas sadias.

Desde 1970 os perfis metabólicos são empregados para o monitoramento rotineiro como meio de diagnóstico das doenças metabólicas nutricionais e como medidas de diagnóstico dos transtornos subclínicos. Por meio das análises sanguíneas de grupos representativos de animais de um rebanho é possível estabelecer seu grau de adequação nas principais vias metabólicas relacionadas às proteínas, minerais e energia, bem como a funcionalidade de órgãos vitais para a produção de leite, como é o caso do fígado (WITTWER, 2000; DUFFIELD e LEBLANC, 2009). 
A interpretação do perfil metabólico requer uma avaliação cuidadosa tanto quanto aplicada a rebanhos como a indivíduos, devido aos mecanismos homeostáticos que controlam o nível sanguíneo dos vários metabólitos e as variações desses níveis em função de fatores como raça, idade, estresse, dieta, nível de produção leiteira, manejo, clima, estado fisiológico, além de dados como o tipo e a quantidade da forragem consumida, história clínica e produtiva do rebanho e o estágio reprodutivo (GONZÁLEZ, 1997). A avaliação do perfil metabólico tem a finalidade de identificar as vacas com risco para enfermidades e assim evitar ou atenuar o problema clínico (LEBLANC et al., 2006).

\section{Objetivos}

O objetivo deste trabalho foi estabelecer o perfil metabólico de magnésio, cálcio, fósforo, triglicerídeos e glicose de vacas leiteiras da raça holandesa PO (Puro de Origem) durante o período de transição, clinicamente hígido.

\section{Material e Métodos}

O experimento foi realizado na Fazenda Pindobas, Venda Nova do Imigrante, Espírito Santo, Brasil. Foram coletadas amostras de $(4 \mathrm{~mL})$ de sangue de 56 vacas leiteiras de alta produção da raça Holandesa PO (Puro de Origem), hígidos ao exame físico. A produção diária das vacas utilizadas no experimento era uma média de 25 a $30 \mathrm{~kg} /$ dia de leite. Os animais são criadoss em sistema de confinamento do tipo free-stall e alimentadas com silagem de milho misturada com ração balanceada ${ }^{1}$ pelo médico veterinário da fazenda, feno de capim tifton (Cynodon dactylon), sal mineral ${ }^{2}$ e água ad libitum. As vacas em

\footnotetext{
${ }^{1}$ Ração coopeavi leite

${ }^{2}$ Premix para vaca de leite
} 
lactação recebem ração diariamente que contém $27 \%$ de proteína bruta (PB), já as vacas do pré-parto recebem ração pré-parto com $24 \%$ de PB. Para as vacas do pré-parto não é fornecido sal mineral. As amostras foram coletadas em cinco momentos: 30 e 15 dias pré-parto, no dia do parto; 15 e 30 dias após o parto. A coleta foi realizada após a ordenha da manhã. Todas as amostras forma obtidas mediante punção venosa coccígea em tubos a vácuo ${ }^{3}$ de $10 \mathrm{~m} \mathrm{~mL}$ sem anticoagulante, sendo uma sendo uma fração dessa amostra utilizada para dosagem imediata de glicose com o auxílio do glicosímetro de uso humano ${ }^{4}$, em seguida o soro foi extraído após a sedimentação, sendo armazenadas em tubos de eppendorf a temperatura de $-20^{\circ} \mathrm{C}$ até o processamento. Não foram utilizados os mesmos animais nos diferentes momentos, portanto para a determinação dos momentos do pré-parto foi utilizada a tabela da data da inseminação artificial, os demais momentos foram determinados por meio do registro da data de parto. O número de vacas utilizadas em cada um dos cinco momentos está descrito na tabela 1.

Tabela 1: Número de animais relacionados aos momentos de coleta

\begin{tabular}{lc}
\hline \multicolumn{1}{c}{ Momentos de coletas } & Número de animais \\
\hline Trinta dias pré-parto (M1) & 13 \\
Quinze dias pré-parto (M2) & 14 \\
Parto (M3) & 8 \\
Quinze dias pós-parto (M4) & 8 \\
Trinta dias pós-parto (M5) & 13 \\
\hline Total & $\mathbf{5 6}$ \\
\hline
\end{tabular}

As amostras foram encaminhadas para o laboratório clínico (CEMEVES). As dosagens de triglicerídeos, cálcio, fósforo e magnésio foram realizadas de acordo com a técnica recomendada pelos kit's comerciais ${ }^{5}$ e determinadas em

\footnotetext{
${ }^{3}$ vacutainer ${ }^{\circledR}$

${ }^{4}$ Optium Exced ${ }^{\circ}$

${ }^{5}$ Labteste ${ }^{\circledR}$
} 
aparelho de espectrofotometria ${ }^{6}$. A análise estatística foi feita utilizando o teste T de Student, para comparação entre os momentos pré e pós-parto em relação ao momento do parto, com nível de significância de $5 \%$.

\section{Resultados e Discussão}

À análise estatística, para dosagem bioquímica de cálcio constatou-se diferença significativa entre os momentos 30 dias do pré-parto (M1) e o parto (M3) (Tabela 2). O aumento dos valores de cálcio nesta fase pode ser decorrente da modificação do manejo nutricional adotado na fazenda. As vacas a partir dos 30 dias do pré-parto não ingerem mais sal mineral, reduzindo, portanto a ingestão de cálcio.

Tabela 2. Níveis séricos de cálcio, fósforo e magnésio em vacas no período de transição

\begin{tabular}{lcccc}
\hline \multirow{2}{*}{ Momentos de coleta } & No de & \multicolumn{3}{c}{ Níveis séricos } \\
\cline { 3 - 5 } & animais & $\begin{array}{c}\text { Cálcio } \\
(\mathrm{mg} / \mathrm{dL})\end{array}$ & $\begin{array}{c}\text { Fósforo } \\
(\mathrm{mg} / \mathrm{dL})\end{array}$ & $\begin{array}{c}\text { Magnésio } \\
(\mathrm{mEq} / \mathrm{L})\end{array}$ \\
\hline $\begin{array}{l}\text { Trinta dias pré-parto } \\
\text { (M1) }\end{array}$ & 13 & $9,12 \pm 1,04^{*}$ & $7,52 \pm 1,27$ & $1,94 \pm 0,39 *$ \\
$\begin{array}{l}\text { Quinze dias pré-parto } \\
\text { (M2) }\end{array}$ & 14 & $11,72 \pm 1,29$ & $7,91 \pm 1,18$ & $2,54 \pm 0,50^{*}$ \\
$\begin{array}{l}\text { Parto } \\
\text { (M3) }\end{array}$ & 8 & $11,56 \pm 0,99$ & $7,44 \pm 1,37$ & $3,08 \pm 0,56$ \\
$\begin{array}{l}\text { Quinze dias pós-parto } \\
\text { (M4) }\end{array}$ & 8 & $8,40 \pm 1,73^{*}$ & $7,11 \pm 1,07 *$ & $2,16 \pm 0,53^{*}$ \\
$\begin{array}{l}\text { Trinta dias pós-parto } \\
\text { (M5) }\end{array}$ & 13 & $11,62 \pm 0,89$ & $7,54 \pm 1,00$ & $2,81 \pm 0,39$ \\
\hline
\end{tabular}

*Médias nas colunas diferem estatisticamente dos valores do parto.

Esse achado vem de encontro com as informações descritas por Santos e Santos (1998), NRC (2001), Schafhauser Jr (2006) os quais afirmam que a

\footnotetext{
${ }^{6}$ Bio 200 da BioPlus ${ }^{\circledR}$
} 
baixa ingestão de cálcio leva a ativação do sistema homeostático deste elemento, e estimula a retirada de cálcio das reservas ósseas, elevando os níveis séricos. Esse procedimento nutricional é importante para reduzir a incidência de hipocalcemia da parturiente.

Através da análise verificou-se o menor valor de cálcio sérico no M4, de 8,4 $\mathrm{mg} / \mathrm{dL}$, provavelmente devido a alta produção de leite após o parto que demanda altas quantidades desse mineral, visto que as vacas produzem em média 25 a 30 Kg/leite/dia (GOFF, 2006), considerado por Goff e Horst (1997) como hipocalcemia subclínica, no entanto sendo resultado dentro de valores de normalidade citados por Rosol e Capen (1997), e acima dos valores citado para diagnostico de hipocalcemia subclinicos, como afirmado por Duffield e Lablanc (2009) (Tabela 2).

Os valores encontrados após o parto divergem dos relatados por Leblanc et al. (2005) e Frigotto et al. (2009), os quais encontraram valores de 11,22 mg/dL no décimo dia após o parto, essa diferença pode ser justificada pela alta produção leiteira dos animais utilizados na pesquisa.

No (M5), não houve relevância estatística, e o valor encontrado apresentavase dentro dos valores de referência citados por Rosol e Capen (1997), o que significa que apesar do momento de grande produtividade a dieta ministrada ao animal consegue suprir as necessidades para mantença e produção (NRC, 2001).

Os valores de fósforo encontrados estavam de acordo com os determinados por Goff (2006), onde a média mais baixa foi durante o período de transição. O único resultado que apresentou relevância foi o (M4), momento o qual a vaca apresenta em fase lactante, e que demanda um consumo aumentado deste mineral (RADOSTITS et al., 2000). 
Os valores de magnésio mais baixos de significância estatística foi de 1,94 $\mathrm{mEq} / \mathrm{L}$, ao $M 1$, mas se apresenta dentro dos valores de normalidade citados por Goff (2006) e Radostits et al. (2000) onde as concentrações séricas de magnésio variam de 1,7 a $3 \mathrm{mEq} / \mathrm{L}$, sendo assim é visível que a nutrição feita para os animais estavam suprindo as necessidades e com isso inibindo efeitos indesejados como citados por (GOFF, 2000; NRC, 2001).

O decréscimo na concentração sérica de cálcio, fósforo e magnésio aos (M4) pode ter sido conseqüência da alta produção de leite que varia em torno de 25 a $30 \mathrm{Kg} / \mathrm{dia}$. Corroborando com dados descritos por Goff e Horst, (1997). Radostits et al. (2000), Goff (2006) ressaltam que ocorre essa alteração devido a redução dos níveis séricos de cálcio que é demandado para a alta produção de leite, com isso ocorre o estímulo para a liberação de PTH, que promove a diminuição dos níveis séricos de fósforo devido ao aumento da excreção renal e salivar do fósforo.

Estatisticamente observou-se variação dos níveis séricos de glicose nos momentos M1, M2, M4 e M5 (Tabela 3). A variação do M1 e M2 pode estar associada variações físicas sofrida pelo animal, como diminuição da capacidade ruminal, associado ao momento do crescimento fetal (CHEW et al., 1979).

Tabela 3. Níveis séricos de triglicerídeos e glicose

\begin{tabular}{lccc}
\hline \multirow{2}{*}{ Momentos de coleta } & \multirow{2}{*}{$\begin{array}{c}\text { No de } \\
\text { animais }\end{array}$} & \begin{tabular}{c} 
Níveis \\
\cline { 3 - 4 }$(\mathrm{mg} / \mathrm{dL})$
\end{tabular} & Glicose $(\mathrm{mg} / \mathrm{dL})$ \\
\hline $\begin{array}{l}\text { Trinta dias pré-parto } \\
\text { (M1) }\end{array}$ & 13 & $32,92 \pm 10,32^{*}$ & $49,00 \pm 8,65^{*}$ \\
$\begin{array}{l}\text { Quinze dias pré-parto } \\
\text { (M2) }\end{array}$ & 14 & $25,71 \pm 6,85^{*}$ & $56,64 \pm 12,46^{*}$ \\
$\begin{array}{l}\text { Parto (M3) } \\
\begin{array}{l}\text { Quinze dias pós-parto } \\
\text { (M4) }\end{array}\end{array}$ & 8 & $14,63 \pm 4,47$ & $73,75 \pm 21,63$ \\
$\begin{array}{l}\text { Trinta dias pós-parto } \\
\text { (M5) }\end{array}$ & 13 & $20,75 \pm 3,88^{*}$ & $60,00 \pm 25,28^{*}$ \\
\hline
\end{tabular}

*Médias nas colunas diferem estatisticamente dos valores do parto. 
CUPERTINO, C.F. et al. Avaliação do perfil metabólico em vacas leiteiras de alta produção no período de transição. PUBVET, Londrina, V. 5, N. 18, Ed. 165, Art. 1115, 2011.

No entanto se compararmos os níveis de glicose encontrado no presente trabalho com os citados por Kaneko (1997), que considera os valores de normalidade de glicose em torno de 45 a $75 \mathrm{mg} / \mathrm{dL}$, os animais não apresentam alterações fisiológicas significativas, mas ao compararmos com o momento referente ao parto M3, estatisticamente observamos relevância.

Essa variação encontrada entre M1, M2 com o M3 é justificada pelo estresse no momento do parto, caracterizado pelos altos níveis de glicocorticóides, onde há uma liberação de cortisol, que por sua vez é um hormônio hiperglicemiante, além de estar associado ao aumento do glucagon que acarretam na redução dos estoques de glicogênio no fígado (GRUMMER, 1995).

Os momentos M4 e M5 houve diferenças significativas com o M3, provavelmente houve essa diminuição, devido a maior ingestão de alimentos pela vaca logo após o parto, uma vez que sua capacidade de ingestão vai aumentando gradativamente, devido a involução uterina e distensão ruminal (VASQUEZ-ANON et al., 1994).

$\mathrm{Na}$ avaliação dos triglicerídeos foram encontradas variações entre, M1, M2 quando relacionados ao $\mathrm{M} 3$, onde os valores desses primeiros momentos estavam aumentados quando comparado ao momento do parto, pois durante essa fase do período de transição é grande a demanda de energia e por isso o organismo faz uso de sua reserva lipídica denominada lipomobilização, que acarreta a liberação de triglicerídeos e, portanto aumento dos níveis séricos (SMITH et al., 1997; BOBE et al., 2004, DUFFIELD e LEBLANC, 2009).

Em alguns momentos se comparados os valores numéricos como no caso do M4, os níveis de triglicerídeos estão diminuídos em relação aos momentos anteriores M1 e M2, provavelmente devido às concentrações plasmáticas de glicose mais elevadas suprindo a demanda energética reduzindo a lipomobilização, como destacado por Grumer (2009). Vasquez-Anon et al. 
(1994) e González et al. (1996) relataram que nos momentos pré-parto a glicemia diminui um pouco ou permanecem estáveis, pois ocorre uma maior demanda de energia para o desenvolvimento do feto além da demanda de glicose pela glândula mamária para a produção de colostro, como foi constatado nesse trabalho.

Após o parto no M4, os níveis de triglicerídeos aumentaram em relação ao parto, provavelmente devido à alta produção de leite no início da lactação, que demanda uma grande quantidade de energia, que leva a lipomobilização das reservas corporais da vaca (SMITH et al., 1997)

\section{Conclusão}

Através dos resultados obtidos no presente trabalho conclui-se, que a dosagem bioquímica de cálcio, fósforo, magnésio, triglicerídeos e glicose podem ser utilizados como ferramenta para o monitoramento do perfil metabólico, de vacas leiteiras no período de transição, assim como para orientação dos Médicos Veterinários e Produtores quanto ao sistema de manejo empregado.

Foi possível verificar, que mesmo os animais sendo hígidos, nos diferentes momentos avaliados houve oscilação nos parâmetros, entretanto estes se mantiveram dentro dos valores de referências.

Para a prevenção das doenças metabólicas é fundamental um manejo nutricional adequado de acordo com a fase produtiva, dos animais. Visto que o período de transição é o momento mais crítico das vacas leiteiras de alta produção. 


\section{Referências Bibliográficas}

AMETAJ, B. N. A new understanding of the causes of fatty liver in dairy cows. Advances in Dairy Technology, Canada, v. 17, p. 97-112, 2005.

BOBE, G.; YOUNG, J. W.; BEITZ, D. C. Invited review: pathology, etiology, prevention, and treatment of fatty liver in dairy cows. Journal of Dairy Science, Iowa, v. 87, p. 3105- 310, 2004.

CHEW, B. P.; ERB, R. E.; FESSLER, J. F.; CALLAHAN, C. J.; MALVEN, P. V. Effects of ovariectomy during pregnancy and of prematurely induced parturition on progesterone, estrogens, and calving traits. Journal of Dairy Science, Lafayette, v. 62, p. 557-566, 1979.

CARVALHO L. A.; NOVAES L. P.; MARTINS C. E.; ZOCCAL R.; MOREIRA P.; RIBEIRO A.C. C. L; LIMA V. M. B. Sistema de Produção de Leite (Cerrado). Embrapa gado de leite 2002; Disponível em: <http://www.sistemasdeproducao.cnptia.embrapa.br/leite/leitecerrado.html>. Acesso em 20 mar. 2010.

DUFFIELD, T. F.; LEBLANC, S. J. Interpretation of serum metabolic parameters around the transition period. Southwest Nutrition and Management Conference, p. 106-114, 2009. Disponível em: < http://www.ag.arizona.edu/ANS/swnmc/papers/2009/.pdf>. Acesso em 20 de mar. 2010.

FRIGOTTO, T. A.; OLLHOFF, R. D.; FILHO, I. R. de BARROS.; ALMEIDA, R. Parâmetros metabólicos sanguíneos de vacas leiteiras de alta produção no período de transição. In: VIII CONGRESSO BRASILEIRO DE BUIATRIA, 8., 2009, Belo Horizonte. Anais do VIII Congresso Brasileiro de Buiatria: Ciência Animal Brasileira, 2009. p. 99-105.

GOFF, J. P.; HORST, R. L. Physiological changes at parturition and their relationship to metabolic disorders. Journal of Dairy Science, Estados Unidos, v. 80, p. 1260-1268, 1997.

GOFF, J. P. Pathophysiology of calcium and phosphorus disorders. The Veterinary Clinics of North America, Iowa, v. 16, p. 319-337, 2000.

GOFF, J. P. Distúrbios do metabolismo dos carboidratos e da gordura. In: REECE, O. W. Dukes: Fisiologia dos animais domésticos. 10. ed. Rio de Janeiro: Guanabara Koogan, 2006. p. 510-515.

GOFF, J. P. Minerais. In: REECE, O. W. Dukes: Fisiologia dos Animais Domésticos. 10. ed. Rio de Janeiro: Guanabara Koogan, 2006. p. 532-540.

GOFF, J. P.; KIMURA, K. Interação entre doenças metabólicas e o sistema imune. In: XIII CURSO NOVOS ENFOQUES NA PRODUÇÃO E REPRODUÇÃO DE BOVINOS, 13., 2009, Uberlândia. Anais do XIII Curso Novos Enfoques na Produção e Reprodução de Bovinos: Centro Nacional de Doença Animal, Uberlândia, 2009, p. 251-261.

GONZÁLEZ, F.; MUINO, R.; PEREIRA, V.; CAMPOS, R.; CASTELLOTE, J. L. B. Indicadores sanguíneos de lipomobilização e função hepática no início da lactação em vacas leiteiras de alta produção. In: VIII Congresso Brasileiro de Buiatria, 6., 2009, Belo Horizonte. Anais do VIII Congresso Brasileiro de Buiatria: Ciência Animal Brasileira. Belo Horizonte, 2009. p. 65-67. 
GONZÁleZ, F. H. D.; HAIDA, K. S.; ZANOLLA, N.; FIGUR, K. Influência da época do ano no perfil metabólico em gado leiteiro no sul do Brasil. Arquivo Faculdade Veterinária Rio Grande do Sul, Porto Alegre, v. 24, n. 2, p. 11-24, 1996.

GONZÁLEZ, F. H. D. O perfil metabólico no estudo de doenças da produção em vacas leiteiras. Arquivo Faculdade Veterinária Rio Grande do Sul, Porto Alegre, v. 25, n. 2., p. 13-33, 1997.

GONZÁLEZ, F. H. D. Indicadores sanguíneos do metabolismo mineral em ruminantes. In: GONZÁleZ, F. H. D.; BARCELlOS, J.; PATINO, H. O.; RIBEIRO, C. A. GONZÁLEZ, F. H. D.; Perfil Metabólico em Ruminantes: Seu uso do perfil metabólico para determinar o status nutricional em gado de corte. Porto Alegre: Gráfica UFRGS, 2000. Cap. 23, p. 8-10.

GRUMMER, R. R. Impact of changes in organic nutrient metabolism on feeding the transition dairy cow. Journal of Animal Science, Madison, v. 73, p. 2820-2833, 1995.

GRUMMER, R. R. Qual período é mais critico: pré-parto vs periparto vs pós-parto. In: XIII CURSO NOVOS ENFOQUES NA PRODUÇÃO E REPRODUÇÃO DE BOVINOS, 13., 2009, Uberlândia. Anais do XIII Curso Novos Enfoques na Produção e Reprodução de Bovinos: Centro Nacional de doença animal, Uberlândia, 2009, p. 237-243.

HERDT, T. Distúrbio e absorção: os processos não fermentativos. In: CUNNINGHAM, J. G. Tratado de Fisiológica Veterinária. 2. ed. Rio de Janeiro: Guanabara Koogan, 1996. Cap. 29 , p. 243-253.

HUZZEY, J. M.; VEIRA, D. M.; WEARY, D. M.; VON KEYSERLINGK, M. A. G. Prepartum behavior and dry matter intake identify dairy cows at risk for metritis. Journal of Dairy Science, Canadian, v. 90, p. 3220- 3233, 2007.

KANEKO, J. J. Carbohydrate metabolism and its diseases. In: KANEKO, J. J.; HARVEY, J. W.; BRUSS, M. L. Clinical Biochemistry of Domestic Animals. 5. ed. San Diego: Academic Press, 1997. Cap. 3, p. 45-68.

KOZLOSKI, G. V. Metabolismo intermediário. In: Bioquímica dos ruminantes. 1. ed. Santa Maria: UFSM, 2002. Cap. 4, p.123-135.

LEBLANC, S. J.; LISSEMORE, K. D.; KELTON, D.F.; T. F. DUfFIELD, T. F.; LESLIE, K. E. Major advances in disease prevention in dairy cattle. Journal of Dairy Science, Canadian, v. 89, p. 1267-1279, 2006.

LEBLANC, S. J.; LESLIE, K.; DUFFIELD, T. F. Metabolic predictors of displaced abomasum in dairy cattle. Journal of Dairy Science, Canadian, v. 88, p. 159-170, 2005.

NIELEN, M.; AARTS, M. G. A.; JONKERS, A. G. M.; WENSING, T.; SCHUKKEN, Y.H. Evaluation of two cow side tests for the detection of subclinical ketosis in dairy cows. Canadian Veterinary Journal, Ottawa, v.35, p. 229-232, 1994.

NRC. National reserch council. Nutrient requirements of dairy cattle, 2001. Disponível em: <http://www.books.nap.edu/openbook.>. Acesso em: 26 abr. 2010.

PEIXOTO, P. V.; MALAFAIA, P.; BARBOSA, J. D.; TOKARNIA, C. H. Princípios de suplementação mineral em ruminantes. Pesquisa Veterinária Brasileira, Rio de Janeiro, v. 25, n.3., p. 195200, 2005. 
PETTERSON, J. A.; DUNSHEA, F. R.; EHRHARDT, R. A.; BELL, A. W. Pregnancy and clndernutrition alter glucose metabolic responses to insulin in sheep. Journal of Nutrition, Ithaca, p. 1286-1295, 1993.

RADOSTITS, M. O. ; GAY, C. C.; BLOOD, C. D.; HINCHCLIFF, W. K. Doenças metabólicas. In: Um tratado de doenças dos bovinos, ovinos, suínos, caprinos e equinos. 9. ed. Rio de Janeiro: Guanabara Koogan, 2000. Cap. 28, p. 1275 - 1324.

RODRIGUES, R.; GONZÁLEZ, F. H. D. Distúrbios do metabolismo do cálcio: hipocalcemia puerperal e eclampsia. Rio Grande do Sul, 2004. 12p. Dissertação (Pós-graduação) Universidade Federal do Rio Grande do Sul, Universidade Federal do Rio Grande do Sul.

ROSOL, J. T.; CAPEN, C. C. Calcium-regulating hormones and diseases of abnormal mineral (calcium, phosphorus, magnesium) metabolism. In: KANEKO, J. J.; HARVEY, J. W.; BRUSS, M. L. Clinical Biochemistry of Domestic Animals. 5. ed. San Diego: Academic Press, 1997. Cap.23, p. 620-685.

SANTOS, J. E. P.; SANTOS, F. A. P. Novas estratégias no manejo e alimentação de vacas préparto. In: X SIMPÓSIO DE PRODUÇÃO ANIMAL, 10.,1998, São Paulo. 1998. p. 165- 214.

SCHAFHAUSER JR, J. O balanço de cátions e ânions em dietas para vacas leiteiras no período de transição. Revista da Faculdade de Zootecnia, Veterinária e Agronomia, Uruguaiana, v. 13, n.1., p. $112-127,2006$.

SMITH, T. R.; HIPPEN, A. R.; BEITZ, D. C.; YOUNG, J.W. Metabolic characteristics of induced ketosis in normal and obese dairy cows. Journal of Animal Science, Iowa, v. 80, p $1569-$ 1581,1997 .

VASQUEZ-ANON, M.; BERTICS, S.; LUCK, M.; GRUMMER, R.R Peripartum liver triglyceride and plasma metabolites in dairy cows. Journal of Dairy Science, Madison, v. 77, p. 1521-1528, 1994.

WEST, H.J. Effect on liver function of acetonemia and the fat cow syndrome in cattle. Research in Veterinary Science, London, v. 48, p 221-227, 1990.

WITTWER M. M. V. Diagnóstico dos desequilíbrios metabólicos de energia em rebanhos bovinos. In: GONZÁleZ, F. H. D.; BARCELloS, J.; PATINO, H. O.; RIBEIRO, C. A. Perfil Metabólico em Ruminantes: Seu Uso em Nutrição e Doenças Nutricionais. Porto Alegre: Gráfica UFRGS, 2000. Cap. 31 , p. 23-24 